\title{
Guest editorial: noncoding RNA in hematopoietic system
}

\author{
Ai Kotani
}

Received: 20 March 2014/Accepted: 25 March 2014/Published online: 4 April 2014

(c) The Japanese Society of Hematology 2014

Following the completion of the Human Genome Project, which revealed that the number of the protein-coding genes in human and mouse is nearly the same, many researchers began to be interested in non-coding RNAs, which are transcribed from so-called "junk DNA", a name suggesting these are no more than genomic garbage. This genomic region does not code protein, and shows considerable interspecies and inter-individual diversity, suggesting that it has the potential to be key to explaining the difference between human and mice, and between human individuals. Early studies focused on small RNAs including miRNAs, endo siRNAs, and piRNA, but more recently, the biological significance of long non-coding RNAs has begun to come into focus.

In the hematopoietic system, Chang-Zheng Chen and Harvey Lodish first demonstrated that miR-181 affects B cell fate in 2004 [1]. Klaus Rajewsky subsequently established miR-155 deficient mice in 2006, showing that miR155 is critically important in B cell development and activation [2]. A body of work that accumulated thereafter clearly revealed that miRNAs are involved in this and other cellular biological systems. The study of long non-coding RNAs in the hematopoietic system thus remains in its early stages. However, a number of recent studies have yielded exciting new insights.

This issue includes two excellent review articles [3, 4], in addition to one by our own groups [5]. Ikeda et al. who have long focused on the function of miRNAs in hematological malignancy, thoroughly review studies of miRNAs in $\mathrm{T}$ cell malignancy, including their recent new findings,

\footnotetext{
A. Kotani $(\bowtie)$

Department of Hematology and Oncology, Tokai University

School of Medicine, Kanagawa, Japan

e-mail: aikotani@k-lab.jp
}

as well as areas needing further investigation. This promises to be one of the most informative reviews on this important topic.

The biological significance of long non-coding RNAs has long remained hidden due in part to its poor conservation. For the first time, Alvarez-Dominguez et al. [6] describe a number of characteristic features, including developmental stage-specific expression profiles, which suggest that long non-coding RNAs potentially have important biological functions in the hematopoietic system. The group, a leader in this field, reviews and discusses recent findings of long non-coding RNAs in hematopoietic system, with a special focus on phenotypes made possible by gene targeting technology, giving important new insights into the roles played by long non-coding RNAs in the hematopoietic system. Although still in its early days, this area of inquiry shows tremendous promise for the future.

In the final review, our group focuses on recent developments involving cell fate-related miRNAs, non-canonical small RNAs, and secretory small RNAs in hematopoietic system. I hope that this month's "Progress in Hematology" will help readers to understand recent progress in our understanding of the biological significance of noncoding RNAs in the hematopoietic system.

\section{References}

1. Chen CZ, Li L, Lodish HF, Bartel DP. MicroRNAs modulate hematopoietic lineage differentiation. Science. 2004;303(5654): 83-6.

2. Thai T, Calado D, Casola S, et al. Regulation of the germinal center response by microRNA-155. Science. 2007;316(5824):604-8.

3. Alvarez-Dominguez JR, Hu W, Gromatzky AA, Lodish HF Long Noncoding RNAs during Normal and Malignant Hematopoiesis. Int J Hematol. 
4. Ikeda S, Tagawa $H$ Dysregulation of microRNAs and their association in the pathogenesis of T-cell lymphoma/leukemias. Int J Hematol.

5. Okuyama K, Ogata J, Yamakawa N, Chanda B, Kotani AI Small RNA as a regulator of hematopoietic development, immune response in infection and tumorigenesis. Int J Hematol.
6. Alvarez-Dominguez JR, Hu W, Yuan B, Shi J, Park SS, Gromatzky AA, van Oudenaarden A, Lodish HF. Blood. 2014;123(4):570-81. 